\title{
CÁLCULO DE DANO AMBIENTAL DECORRENTE DE UM DEPÓSITO DE LIXO: ESTUDO DE CASO
}

\author{
Josimar Ribeiro de Almeida \\ Observatório Urbano do Estado do Rio de Janeiro - OUERJ/UERJ/UN-Habitat \\ $\bowtie$ jralmeida@usp.br \\ Gustavo Aveiro Lins \\ CEDERJ/ Mestre em Engenharia -Professor SEE/RJ/Observatório Urbano Estado do Rio de Janeiro- \\ OUERJ/UERJ/UN-Habitat \\ Elenice Rachid \\ Mestranda em Engenharia de Transporte - PET/COPPE/UFRJ. Pesquisadora UFRJ - NASA (CNPq)
}

\section{Resumo \\ O presente trabalho teve como foco principal aplicar uma metodologia capaz de estimar o dano ambiental decorrente de atividades antrópicas. Neste artigo foi utilizado como modelo a instalação de um depósito de lixo. \\ Palavras-chave: dano ambiental, impactos ambientais, economia ambiental \\ CALCULATION OF ENVIRONMENTAL DAMAGE ARISING AT THE JUNKYARD: CASE STUDY}

\begin{abstract}
The present work had as main focus apply a methodology able to estimate the environmental damage resulting from antropic activities. This article was used as a template to install a junkyard.
\end{abstract}

Keywords: environmental damage, environmental impacts, environmental economics

\section{INTRODUÇÃO}

O presente trabalho foi elaborado a partir de uma análise pericial, cujo foco principal foi estimar o dano ambiental decorrente da instalação de um depósito de lixo na região. As partes envolvidas neste estudo de caso são: o município como órgão responsável pela instalação do depósito de lixo e uma cooperativa de agricultores que se sentiram prejudicados por esta iniciativa do município.

O Objetivo deste trabalho é descrever a metodologia utilizada para calcular o dano ambiental na referida situação. 


\section{METODOLOGIA}

O dano ambiental foi dividido em dano a produção agrícola e dano à água. Para efeito de desvalorização do terreno supomos que o dano ambiental calculado inclui as perdas de desvalorização e produção. Para efeito de produção foi considerada a perda de produção por oportunidade ou Custo de Oportunidade, pelo fato de não poder fazer.

O método utilizado para o cálculo dos danos ambientais à produção agrícola foi feito para o dano a produção vinífera existente e para o custo de oportunidade de produção de viníferas. Salienta-se que o custo de oportunidade é passível de cálculo neste caso, pois a região é de aptidão para o cultivo de viníferas. O valor médio de produção é de $1.698,83 \mathrm{~kg} / \mathrm{ha}$, baseado nos dados do município. O valor encontrado é a divisão de produção média de $43.300 .000 \mathrm{~kg} / \mathrm{ano}$ pela área do município que é de $254,88 \mathrm{~km} 2$.

Para o cálculo dos danos ambientais à água, o método usado é aproximações semiquantitativas ou também chamado de Lista de Verificação Ponderada. Neste método se avalia o Índice de Qualidade Ambiental (IQA) a partir das amostras coletadas. Os parâmetros do CONAMA serão usados sempre que possível para o índice de qualidade de $100 \%$ e, da bibliografia para $0 \%$. Estes limites foram usados como valores limites de interpolação. Com o valor do dano estimado, é calculado o valor dos Custos Ambientais Totais Esperados (CATE) para os danos intermitentes e contínuos.

\section{CÁlCULO DO DANO AMBIENTAL NA PRODUÇÃO DE VINÍFERAS}

\section{Características da plantação existente:}

- Área plantada: 4 ha

- Produtividade média: $1698,83 \mathrm{~kg} / \mathrm{ano}$

- Valor de venda: 0,28 R \$/kg (Fonte: Jornal Gazeta Mercantil)

\section{Caso sem o depósito de lixo:}

- Custo de produção: $50 \%$ do preço de venda $=0,28 \times 0,50=0,14 \mathrm{R} \$ / \mathrm{kg}$

- Custo de formação: $50 \%$ do custo de produção $=0,14 \times 0,50=0,07 \mathrm{R} \$ / \mathrm{kg}$

- Custo de colheita:33\% do custo de produção $=0,14 \times 0,33=0,047 \mathrm{R} \$ / \mathrm{kg}$

- Lucro bruto: $50 \%$ do preço de venda $=0,28 \times 0,50=0,14 \mathrm{R} \$ / \mathrm{kg}$ 


\section{Caso com o depósito de lixo:}

- Custo de produção: $50 \%$ do preço de venda + diferença pelo uso de mais defensivo agrícola

- Custo de formação: 0,07 $\mathrm{R} \$ / \mathrm{kg}$ x 1,4 (aumento de custo) = 0,098 $\mathrm{R} \$ / \mathrm{kg}$ (foi considerado um aumento de $40 \%$ no custo de formação pelo uso de mais defensivos agrícolas e mão de obra na aplicação)

- Diferença entre o custo normal de formação e manutenção e o custo de formação e manutenção após a instalação do aterro de lixo $=0,098-0,070=0,028 \mathrm{R} \$ / \mathrm{kg}$

\section{Dano previsto na produção de viníferas:}

- Dano unitário: 0,028 R \$/kg x 1698,83 kg/ha . ano = 47,56 R \$/ ha . ano

\section{Custo de Oportunidade (CO)}

$\mathrm{CO}=1698,33 \mathrm{~kg} / \mathrm{ha}$. ano $\mathrm{x} 0,14 \mathrm{R} \$ / \mathrm{kg}=237,83 \mathrm{R} \$ / \mathrm{ha}$. ano

Impostos $=23,78 \mathrm{R} \$ / \mathrm{ha}$. ano

Custo de Oportunidade Líquido = 214,05 R $\$ /$ ha . ano

Não foram consideradas as outras culturas, devido ao fato de ter sido calculado o Custo de Oportunidade para a área onde não existem viníferas plantadas. Observa-se que o $\mathrm{CO}$ e o dano às viníferas foram calculados baseados em índices de produtividade média por hectare, que normalmente é abaixo do que uma propriedade produz. Isto se deve ao fato de que, da área do município, deveria ser descontado a área urbana, estradas, galpões, residências rurais, florestas, etc. Portanto, o valor é bastante conservativo.

\section{Dano Direto Total na Produção de Viníferas:}

Cd viníferas + CO viníferas = 47,56 R \$/ha.ano x 4 ha +214,67 R \$/ha.ano x 16 há (área plantada $)=3.624,96 \mathrm{R} \$ /$ ano

\section{Cálculo do dano ambiental à água}

O Método escolhido para calcular o dano a partir do Índice de Qualidade Ambiental (I Q A) foi o método de aproximação semi-quantitativo - Lista de Verificação Ponderada. 
Tabela 1 - Determinação das unidades paramétricas de importância (UPI)

\begin{tabular}{lcc}
\hline Parâmetro Analisado & Fim da Pilha (UPI) & Córrego na Propriedade (UPI) \\
\hline $\mathrm{pH}$ & 10 & 4 \\
$\mathrm{DBO}_{5}$ & 2 & 4 \\
Matéria Orgânica & 2 & 4 \\
Nitrogênio Amoniacal & 2 & 4 \\
Nitrito + Nitrato & 2 & 4 \\
Arsênio & 20 & 10 \\
Chumbo & 15 & 10 \\
Cromo Total & 10 & 5 \\
Cádmio & 3 & 5 \\
Mercúrio & 20 & 20 \\
Níquel & 2 & 5 \\
Vanádio & 2 & 5 \\
Coliformes Totais - & - & - \\
Coliforme Fecais & 10 & 20 \\
\hline TOTAL & 100 & 100 \\
\hline
\end{tabular}

Tabela 2 - Determinação do (IQA) 0 - 100\%, para água potável

\begin{tabular}{lcccc}
\hline \multicolumn{1}{c}{ Parâmetro Analisado } & IQA = 0\% & Referência & IQA =100\% & Referência \\
\hline $\mathrm{pH}$ & $10<\mathrm{pH}<4$ & VPM & 7 & Neutra \\
$\mathrm{DBO}_{5}$ & 10 & VPM & $3 \mathrm{mg} / \mathrm{l}$ & Classe 1 \\
Matéria Orgânica & Ausente & VPM & Ausente & \\
Nitrogênio Amoniacal & $0,08 \mathrm{mg} / \mathrm{l}$ & VPM & $0,02 \mathrm{mg} / \mathrm{l}$ & GWQ \\
Nitrito + Nitrato & $10,5 \mathrm{mg} / \mathrm{l}$ & VPM & $10 \mathrm{mg} / 1$ & Classe 1 \\
Arsênio & $0,10 \mathrm{mg} / 1$ & VPM & $0,05 \mathrm{mg} / 1$ & Classe 1 \\
Chumbo & $0,10 \mathrm{mg} / 1$ & VPM & $0,03 \mathrm{mg} / 1$ & Classe 1 \\
Cromo Total & $0,05 \mathrm{mg} / 1$ & VPM & $0,04 \mathrm{mg} / 1$ & GWQ \\
Cádmio & $0,10 \mathrm{mg} / 1$ & VPM & $0,01 \mathrm{mg} / 1$ & Classe 1 \\
Mercúrio & $0,002 \mathrm{mg} / 1$ & VPM & $0,0002 \mathrm{mg} / 1$ & Classe 1 \\
Níquel & $0,05 \mathrm{mg} / 1$ & VPM & $0,025 \mathrm{mg} / 1$ & Classe 1 \\
Vanádio & $0,10 \mathrm{mg} / 1$ & GWQ & $0,1 \mathrm{mg} / 1$ & Classe 1 \\
Coliformes Totais & 1000 & CONAMA & Ausente & \\
Coliforme Fecais & 200 & CONAMA & ausente & \\
\hline
\end{tabular}

VPM - Valores máximos permissíveis; GWQ - A Guide to Water Quality -

FONTE:Agência EPA Canadá, 1979; Classe 1 - RESOLUÇÃO CONAMA 


\section{CÁlCULO do ÍNDICE DE QUALIDAdE DA ÁGUA POTÁVEL E PARA DESSENDENTAÇÃO ANIMAL}

Tabela 3 - Para Água Potável

\begin{tabular}{|c|c|c|c|}
\hline Parâmetro Analisado & $\mathrm{IQA}=0 \%$ & IQA $=100 \%$ & Existente \\
\hline $\mathrm{pH}$ & $10<\mathrm{pH}<4$ & 7 & 8,6 \\
\hline $\mathrm{DBO}_{5}$ & $10 \mathrm{mg} / \mathrm{l}$ & $3 \mathrm{mg} / \mathrm{l}$ & 4,0 \\
\hline Nitrogênio Amoniacal & $0,08 \mathrm{mg} / 1$ & $0,02 \mathrm{mg} / 1$ & 1,1 \\
\hline Nitrito + Nitrato (N2 Total) & $10,5 \mathrm{mg} / 1$ & $10 \mathrm{mg} / \mathrm{l}$ & 1,1 \\
\hline Arsênio & $0,10 \mathrm{mg} / \mathrm{l}$ & $0,05 \mathrm{mg} / \mathrm{l}$ & $\mathrm{ND}^{*}$ \\
\hline Chumbo & $0,10 \mathrm{mg} / \mathrm{l}$ & $0,03 \mathrm{mg} / 1$ & ND \\
\hline Cromo Total & $0,05 \mathrm{mg} / \mathrm{l}$ & $0,04 \mathrm{mg} / 1$ & ND \\
\hline Cádmio & $0,10 \mathrm{mg} / \mathrm{l}$ & $0,01 \mathrm{mg} / \mathrm{l}$ & ND \\
\hline Mercúrio & $0,002 \mathrm{mg} / \mathrm{l}$ & $0,0002 \mathrm{mg} / \mathrm{l}$ & ND \\
\hline Níquel & $0,05 \mathrm{mg} / 1$ & $0,025 \mathrm{mg} / \mathrm{l}$ & ND \\
\hline Vanádio & $0,10 \mathrm{mg} / 1$ & $0,1 \mathrm{mg} / 1$ & ND \\
\hline Coliforme Fecais & 200 & ausente & 930 \\
\hline
\end{tabular}

ND* - Não detectado

Tabela 4 - Para Água de Dessedentação dos Animais

\begin{tabular}{llll}
\hline \multicolumn{1}{c}{ Parâmetro Analisado } & \multicolumn{1}{c}{ IQA $=\mathbf{0 \%}$} & \multicolumn{1}{c}{ IQA =100\% } & Existente \\
\hline $\mathrm{pH}$ & $10<\mathrm{pH}<4$ & 7 & 8,6 \\
$\mathrm{DBO}_{5}$ & $10 \mathrm{mg} / \mathrm{l}$ & $3 \mathrm{mg} / \mathrm{l}$ & 4,0 \\
Nitrogênio Amoniacal & $0,08 \mathrm{mg} / \mathrm{l}$ & $0,02 \mathrm{mg} / \mathrm{l}$ & 1,1 \\
Nitrito + Nitrato (N2 Total) & $10,5 \mathrm{mg} / \mathrm{l}$ & $10 \mathrm{mg} / \mathrm{l}$ & 1,1 \\
Arsênio & $0,10 \mathrm{mg} / \mathrm{l}$ & $0,05 \mathrm{mg} / \mathrm{l}$ & $\mathrm{ND}$ \\
Chumbo & $0,10 \mathrm{mg} / \mathrm{l}$ & $0,03 \mathrm{mg} / \mathrm{l}$ & $\mathrm{ND}$ \\
Cromo Total & $0,05 \mathrm{mg} / \mathrm{l}$ & $0,04 \mathrm{mg} / 1$ & $\mathrm{ND}$ \\
Cádmio & $0,10 \mathrm{mg} / 1$ & $0,01 \mathrm{mg} / 1$ & $\mathrm{ND}$ \\
Mercúrio & $0,002 \mathrm{mg} / \mathrm{l}$ & $0,0002 \mathrm{mg} / \mathrm{l}$ & $\mathrm{ND}$ \\
Níquel & $0,05 \mathrm{mg} / \mathrm{l}$ & $0,025 \mathrm{mg} / \mathrm{l}$ & $\mathrm{ND}$ \\
Vanádio & $0,10 \mathrm{mg} / \mathrm{l}$ & $0,1 \mathrm{mg} / \mathrm{l}$ & $\mathrm{ND}$ \\
Coliforme Fecais & 200 & ausente & 930 \\
\hline
\end{tabular}




\section{CÁlCULO DA QUALIDADE DA ÁGUA POTÁVEL}

A partir das equações de função de qualidade ambiental associadas aos gráficos mostrados na seção de valoração ambiental, temos para cada parâmetro:

\section{pH}

Função: (I Q A)pH = 0,111 pH2 + 1,56 pH - 4,44

Para o valor de $\mathrm{pH}=8,6$, temos (I Q A)pH =0,7156, ou seja, 71,56\%

\section{$\mathrm{DBO}_{5}$}

Função: (I Q A) DBO5 = - $(1 / 22025,2)$ x e DBO5 + 1,00095

Para o valor de DBO5 = 4,0, temos (I Q A) DBO5 = 0,99847, ou seja, 99,84

\section{Nitrogênio Amonical (Namoniacal)}

Função: (I Q A)N amoniacal = - 16,67 Namoniacal + 1,333

Para o valor de Namoniacal = 1,1 é > que 0,02 e 0,08, (I Q A)N amoniacal =0, ou seja, 0\%

\section{Nitrogênio Total $\left(\mathbf{N}_{\mathrm{T}}\right)$}

Função: (I Q A)NT = - 2 NT + 21

Para NT = 1,1 como é < que 20 e 12, (I Q A)NT = 1, ou seja, $100 \%$

\section{Metais Pesados (Ar, PB, Cd, Cr, Hg, Ni, Va)}

Não foram detectados, logo, (I Q A)METAIS = 100\%

\section{Coliformes Fecais (CF)}

Função: (I Q A)CF = - $(1 / 8,984) \times \mathrm{e}-(\mathrm{CF})+1,1113$

Para $\mathrm{CF}=930$, como 930>200, $\log 0(\mathrm{I} \mathrm{Q} A) \mathrm{CF}=0$, ou seja, $0 \%$

\section{CALCULO DO IMPACTO AMBIENTAL (UIA)}

Por definição, UIA = (UPI) $\times($ I Q A)

\begin{tabular}{llll}
\hline Parâmetro Analisado & UPI & I Q A & UIA \\
\hline $\mathrm{pH}$ & 4 & 0,71568 & 2,86272 \\
$\mathrm{DBO}_{5}$ & 4 & 0,99840 & 3,99360 \\
Matéria Orgânica & 4 & 0,00000 & 0,00000
\end{tabular}




\begin{tabular}{llll} 
Nitrogênio Amoniacal & 4 & 1,00000 & 0,00000 \\
Nitrito + Nitrato ( $\mathrm{N}_{2}$ Total) & 4 & 1,00000 & 4,00000 \\
Arsênio & 10 & 1,00000 & 10,00000 \\
Chumbo & 10 & 1,00000 & 10,00000 \\
Cromo Total & 5 & 1,00000 & 5,00000 \\
Cádmio & 5 & 1,00000 & 5,00000 \\
Mercúrio & 20 & 1,00000 & 20,00000 \\
Níquel & 2 & 1,00000 & 2,000000 \\
Vanádio & 3 & 1,00000 & 3,000000 \\
Coliforme Fecais & 25 & 0,00000 & 0,000000 \\
\hline TOTAL & $\mathbf{1 0 0}$ & & $\mathbf{6 5 , 8 5 6 3}$ \\
\hline
\end{tabular}

Logo, a qualidade da água para potabilidade encontrada acima é de 65,85\%, portanto, o dano a água é igual a, DANO A ÁGUA: 1 - 0,6585 = 0,34144, ou seja, 34,141\%

\section{CALCULO dO dANO A ÁGUA POTÁVEL}

* No de pessoas que habitam a propriedade: 7

* Consumo de água por pessoa: 200 litros por dia =0,2 $\mathrm{m} 3 / \mathrm{dia}$

* Consumo diário: 7 pessoas x 0,2 $\mathrm{m} 3 / \mathrm{dia}=1,4 \mathrm{~m} 3 /$ dia

* Consumo anual: 1,4 m3 / dia x 365 dias $=511 \mathrm{~m} 3 /$ ano

Como os índices de Nitrogênio amoniacal e de Coliformes Fecais foram maiores que os valores máximos permitidos, a água é considerada não potável, embora, o seu índice de qualidade tenha sido de $65,85 \%$. Portanto, o dano neste caso é total pois a propriedade não dispõe de sistema de tratamento da água contaminada.

Para a faixa de consumo de $42 \mathrm{~m} 3$ / mês, R \$ 1,77 / m3, fonte Companhia de Água, portanto o dano direto a água será de, Dano Direto a Água Potável = 511 m3/ano x R\$ 1,77 / m3 = 902,77 R\$ / ano ; Cd água potável = 902,77 R\$ / ano

\section{CALCULO DO DANO A ÁGUA DE DESSEDENTAÇÃO E IRRIGAÇÃO}

* No de animais: 15 cabeças de gado, 2 cavalos e 50 galinhas

* Quantidade de água para beber por cabeça de gado = 10 a 15 litros;

por cavalo = 10 a 15 litros;

por galinha $=0,12$ a 1,2 litros. 
* Consumo de água: (15 cabeças x 151 /dia + 2 cavalos x 151 / dia + 50 galinhas x 0,2 1/ dia) $=265$ litros $/ \mathrm{dia}$

*Consumo anual: 2651 / dia x 365 dias $=96,725 \mathrm{~m} 3 /$ ano

* Limpeza de curral/ potreiro/galinheiro = $1001 /$ dia, neste caso não será considerado como dano pois a qualidade da água se presta ao serviço de limpeza.

* Consumo de água sujeita ao dano: 96,725 m3 / ano

Dano Direto Água Dessedentação: 96,725 m3/ano x R \$1,77/m3 = 171,20 R\$/ano

Cd água dess. $=171,20 \mathrm{R} \$ /$ ano

Cd Total da Água $=(902,77+171,20) R \$ /$ ano $=1.073,97$ R $\$$ / ano

\section{CUSTOS DE DANOS JÁ EXISTENTES}

Período considerado: de dezembro de 1992 à abril de 2001 = 8 anos e quatro meses.

Custo dano direto anual: $\mathrm{Cd}$ vinífera+CO viníferas $+\mathrm{Cd}$ água potável+Cd água dess.

Custo do dano direto anual $=47,56 \mathrm{R} \$ /$ ano $\mathrm{x} 4+214,67 \mathrm{R} \$ /$ ano $\mathrm{x} 16+902,77$

$\mathrm{R} \$ /$ ano $+171,20 \mathrm{R} \$ /$ ano $=\mathrm{R} \$ 4.698,93$

\section{CUSTO DO DANO DIRETO ANUAL = R\$ 4.698,93}

Custo do Dano Indireto: Conforme valores apresentados na Tabela 2.4.6 do método CATES, $(\mathrm{Fi} / \mathrm{d})=2$ é um índice razoável, considerando a redução do bem estar da população em relação apenas a custo de dano da água.

\section{CUSTO AMBIENTAL ANUAL}

Custo Ambiental Total $=$ Custo do Dano Total às Viníferas + Custo Total Dano Água Custo Ambiental Total = 3.624,96 R \$ / ano + 1.073,93 R \$ / ano x $2=5.772,82 \mathrm{R} \$ /$ ano Número de anos com degradação na propriedade: 8 anos e 3 meses

DANO AMBIENTAL À PROPRIEDADE ATÉ 29 DE MARÇO DE 2001

$8 \operatorname{anos} x$ 5.700,90 R\$ $/$ ano $=R \$ 47.032,42$

\section{CUSTOS AMBIENTAIS TOTAIS ESPERADOS (CATES)}

Os custos totais esperados são divididos em intermitentes e contínuos.

Caso 1 - Custos Intermitentes 
Supondo que a partir de maio não haverá mais degradação significativa do sitio, ou seja, a usina irá funcionar dentro dos padrões com impacto tolerável, com taxa de juros $4 \%$ ao ano e $\mathrm{n}=25$ anos (uma geração) temos,

$\mathrm{CATE}=\left(\mathrm{V}_{\mathrm{d}}+\mathrm{C}_{\mathrm{d}}-\mathrm{F}_{\mathrm{i} / \mathrm{d}}\right) *(1+\mathrm{J})^{\mathrm{n}} /(1+\mathrm{J})^{\mathrm{n}}-1=(5.772,90 \mathrm{R} \$ /$ ano $\mathrm{x} 2,6658) / 1,6658=\mathrm{R} \$$ $9.238,44$

Caso 2 - Custo Contínuo: Supondo que a partir de maio não entre em operação a usina de lixo, teremos,

CATE $=\left(V_{d}+C_{d}-F_{i / d}\right)\left[(1+J)^{n}-1\right] / j-(1+J)^{n}=(5.772,90 R \$ / a n o) x 1,6658 / 0,1066=R \$$ $90.211,03$

\section{DANO AMBIENTAL TOTAL}

O município mantém em sua administração uma área degradada pela disposição de lixo durante oito anos e quatro meses. O dano ambiental é muito maior que o calculado conforme os quesitos acima. $\mathrm{O}$ balanço social não foi calculado e incluído por não ter sido solicitado. A propriedade avaliada sofreu uma externalidade ambiental, o que significa que alguém tem um benefício e outro sofre um dano. No caso o benefício foi adquirido pela comunidade e o dano foi imposto às pessoas que moram no entorno do aterro de lixo. É importante salientar que este cálculo de dano ambiental foi realizado exclusivamente para a propriedade dos Autores. O dano foi portanto tratado tecnicamente de interesse individual homogêneo, e por isso passível de direito ambiental com a formulação matemática para dano ambiental. Por isto todas as desvalorizações da propriedade já estão inclusas.

Devido a este fato, conclui-se que o dano ambiental total de origem ambiental causado à propriedade dos Autores é o seguinte:

\section{Caso 1 - CATES (intermitente)}

Dano Total $=$ Dano Passado + CATES (intermitente $)$

Dano Total $=\mathrm{R} \$ 47.032,42+\mathrm{R} \$ 9.238,44=\mathrm{R} \$ 56.270,86$

\section{Caso 2 - CATES (contínuo)}

Dano Total $=$ Dano Passado + CATES $($ contínuo $)$

Dano Total $=\mathrm{R} \$ 47.032,42+\mathrm{R} \$ 90.211,03=\mathrm{R} \$ 137.243,45$ 


\section{CONCLUSÃO}

O depósito de lixo da Prefeitura é responsável pelo dano ambiental à área. Os benefícios auferidos pela comunidade não foram avaliados, mas sim o dano no caso existente à propriedade dos autores. A metodologia usada é bastante conservadora, pois foi calculado o dano ambiental sem considerar a valoração de bens de serviço ambiental realizados pela flora e fauna local, que indiretamente beneficiam o ecossistema. Portanto, pode-se com certa segurança afirmar que estes danos seriam os de menor custo. É importante afirmar que o dano calculado à propriedade dos Autores é parte do dano total e não pode ser usado como base para outras propriedades vizinhas e de diferentes variáveis ambientais.

\section{REFERÊNCIAS BIBLIOGRÁFICAS}

ARMAZÉM DE DADOS - INSTITUTO PEREIRA PASSOS. Indicadores de Qualidade Ambiental. Disponível em <http://www.armazemdedados.rio.rj.gov.br/http://portalgeo.rio.rj.gov.br/website/apas/viewer.htm?usu= >, s.d. Acesso em 20 de janeiro de 2013

CONAMA - CONSELHO NACIONAL DE MEIO AMBIENTE. Resolução No 420/2009. Disponível em < http://www.mma.gov.br/conama/ >, s.d. Acesso em 13 de junho de 2013

KOLSTAD, C.D. Environmental Economics. Nova Iorque: Oxford, 2000.

MARTÍNEZ ALIER, J.. The environmentalism of the poor : a study of ecological conflicts and valuation. Northhampton: Edward Elgar Publishing, 2002.

MAY, P. Comércio Agrícola e meio ambiente na América Latina. In: MAY, P.; LUSTOSA, M.C.; VINHA, V.. Economia do Meio Ambiente. Rio de Janeiro: Campus, 2003, pp 197-218.

MAY, P. H.; MOTTA, R.S. (org.). Valorando a Natureza: a análise Econômica para o Desenvolvimento Sustentável. Rio de Janeiro: Campus, 1994.

MAY, P.H. (org.). Economia Ecológica. Rio de Janeiro: Campus, 1995.

MOTTA, R. S. (ed.). Environmental economics and policy making in developing countries : current issues. Cheltenham: Edward Elgar Pub., 2001.

PILLET, G.. Economia ecológica: Introdução à economia do ambiente e dos recursos naturais. Lisboa: Instituto Piaget, 1993. 
ROMEIRO, A.R.; REYDON, B. P; LEORNARDI, M.L.A.. Economia do Meio Ambiente. Campinas: Unicamp, 1997.

SACHS, I.. Caminhos para o desenvolvimento sustentável. Rio de Janeiro: Garamond, 2002.

SERÔA DA MOTTA, R.. Manual de Valoração Econômica de Recursos Ambientais. Brasília: MMA, 1998.

TOMMASI, LR.. Estudos de Impacto Ambiental. São Paulo: Terragraph Artes e Informática, 1994.

WHO - WORLD HEALTH ORGANIZATION.Water pollution control: A guide to the use of water quality

management $\quad$ principles. $\quad$ Disponível em
<http://www.who.int/water_sanitation_health/resources/watpolcontrol/en/index.html>, 1997 Acesso em 09 de outubro de 2013 